\title{
STELLAR IMAGE LOCATION WITH MAXIMUM CORRELATION PROCEDURE
}

\author{
ZHU ZI \\ Shaanxi Astronomical Observatory \\ P.O.Box 18, Lintong, China
}

\begin{abstract}
The location method and accuracy analysis has become a very important subject in astrometry since the photoelectric technique was widely applied. The location methods of the stellar images observed with the slit micrometer are discussed in this paper, and a new estimation method called the maximum correlation procedure is presented. This procedure has already been used to process and analyze the observational data. Because of the ideal mathematical performance of the correlation function, the procedure provides us a good way to determine image locations. By means of simulated observations according to the theoretical analysis, we find that the locating accuracy and the limiting magnitude for this method is much higher than those of traditional locating method. For the observational data of about one thousand stars obtained with the Photoelectric Astrolabe at Yunnan Astronomical observatory, the positional accuracy of a single star using the maximum correlation is improved by 0.045 arcsec over that with the median. The maximum correlation procedure has also increased the data reduction ability for faint star observations with very low signal to noise ratios.
\end{abstract}

\section{References}

Helmer, L., and Morrison, L.V., 1985, Vistas in Astron., 28, 505. Hoeg, E., 1970, Astron. Astrophys., 19, 27.

Li Dongming et al., 1983, Astrolabes and Their Application in Astrometry(in Chinese), Sciences Press, 22.

Lindegren, L., 1978, IAU Coll., 48, 197.

Yoshizawa, M. et al., 1985, Astron. Astrophys., 147, 227.

Yoshizawa, M., and Suzuki, S., 1989, Publ. Natl. Astron. Obs. Japan, 1, 127.

Zhu Zi, 1989, Publ. Shaanxi Astron. Obs., 12, 43. 